\title{
AN INEQUALITY IMPLICIT FUNCTION THEOREM
}

\author{
KUNG-FU NG \\ (Received 3 September 1986; revised 23 February 1987)
}

Communicated by B. Mond

\begin{abstract}
Let $f$ be a continuous function, and $u$ a continuous linear function, from a Banach space into an ordered Banach space, such that $f-u$ satisfies a Lipschitz condition and $u$ satisfies an inequality implicit-function condition. Then $f$ also satisfies an inequality implicit-function condition. This extends some results of Flett, Craven and S. M. Robinson.
\end{abstract}

1980 Mathematics subject classification (Amer. Math. Soc.): 46 A 30, 26 B 10, 46 A 40, 49 B 27.

Following Rockafellar [13], by a convex process is meant a map $T$ of points in a Banach space $X$ into the subsets of another Banach space $Y$ such that $0 \in T 0$, $T(\lambda x)=\lambda T x$ and $T x_{1}+T x_{2} \subseteq T\left(x_{1}+x_{2}\right)$ for all $\lambda>0, x_{1}, x_{2}$ and $x$ in $X$. This is the case if and only if the graph $\mathcal{G}(T)$ of $T$ is a convex cone in $X \times Y$. $T$ is a closed convex process if $\mathcal{G}(T)$ is a closed convex cone. If $T$ is also onto $Y$ (in the sense that for each $y \in Y$ there exists $x \in X$ such that $y \in T x$ ) then it is an open mapping (see [10, Theorem 2] and also [5, page 182], [8, Theorem 1]), that is, there exists a constant $k>0$ with the following property: for each $y \in Y$ there is $x \in X$ with $\|x\| \leq k\|y\|$ such that $y \in T x$. (In this case we say that $T$ is $k$-open.)

Suppose $K$ is a closed convex cone in $Y$. Then, for any continuous linear map $u$ from $X$ into $Y$, we can associate a closed convex process $U$ by putting

$$
U(x)=u(x)+K \quad(x \in X)
$$

Thus, if $U$ is onto $Y$, then $U$ is $k$-open for some $k>0$. The following Theorems 1 and 2 were proved by Flett [4, Lemmas 1 and 3] in the special case that $K=\{0\}$ (see also Craven [2], and [3, page 147]).

(C) 1988 Australian Mathematical Society 0263-6115/88 $\$$ A2.00 + 0.00 
THEOREM 1. Let $U$ be $k$-open for some $k>0$. Let $f$ be a continuous (not necessarily linear) map from a subset of $D$ of $X$ containing 0 into $Y$ such that $f(0)=0$ and

$$
\left\|\left\{f\left(x_{1}\right)-u\left(x_{1}\right)\right\}-\left\{f\left(x_{2}\right)-u\left(x_{2}\right)\right\}\right\| \leq(\eta / k)\left\|x_{1}-x_{2}\right\|
$$

for some $\eta \in(0,1)$ and all $x_{1}, x_{2} \in D$. If $z \in X$ and $D$ contains the ball $B$ with centre $z$ and radius $R$ with $R>(\eta /(1-\eta))\|z\|$, then there exists $x \in B$ such that $u(z) \in f(x)+K$.

The proof is based on the following contraction lemma, essentially due to Robinson [11] who considereci Hausdorff distance $\rho_{H}$ instead of unbalanced $d$ (our proof is also simpler then that given in [11]). See also [7]. For subsets $A$, $B$ of a metric space $(\Omega, \rho)$ and $x \in \Omega$, we define $d(x, B):=\inf \{\rho(x, b): b \in B\}$, $d(A, B):=\sup \{d(a, B): a \in A\}$, and $\rho_{H}(A, B):=\max \{d(A, B), d(B, A)\}$.

LEMMA 1. Let $(\Omega, \rho)$ be a complete metric (or semi-metric) space, and let $T: \Omega \rightarrow 2^{\Omega}$ satisfy

$$
d\left(T x_{1}, T x_{2}\right) \leq \eta \rho\left(x_{1}, x_{2}\right)
$$

for some $\eta \in(0,1)$ and all $x_{1}, x_{2}$ in a subset $D$ of $\Omega$. Suppose $D$ contains a ball $B$ with centre $x_{0}$ and radius $R>d\left(x_{0}, T x_{0}\right) /(1-\eta)$. Then there exists $x \in B$ with $x \in \overline{T x}$.

ProOF. Take $\varepsilon>0$ such that $R>d\left(x_{0}, T x_{0}\right) /(1-\eta)+\varepsilon$, and let $\sigma=$ $d\left(x_{0}, T x_{0}\right)+\varepsilon(1-\eta)$. Since $d\left(x_{0}, T x_{0}\right)<\sigma$, there exists $x_{1} \in T x_{0}$ such that $\rho\left(x_{0}, x_{1}\right)<\sigma$. By $(2)$,

$$
d\left(x_{1}, T x_{1}\right) \leq d\left(T x_{0}, T x_{1}\right) \leq \eta \rho\left(x_{0}, x_{1}\right)<\eta \sigma,
$$

so there is $x_{2} \in T x_{1}$ such that $\rho\left(x_{1}, x_{2}\right)<\eta \sigma$. Suppose that $x_{1}, \ldots, x_{n}$ from $B$ have been selected respectively from $T x_{0}, \ldots, T x_{n-1}$ such that $\rho\left(x_{k-1}, x_{k}\right)<$ $\eta^{k-1} \sigma$ for all $k \leq n$. Then, since

$$
d\left(x_{n}, T x_{n}\right) \leq d\left(T x_{n-1}, T x_{n}\right) \leq \eta \rho\left(x_{n-1}, x_{n}\right)<\eta^{n} \sigma,
$$

one can select $x_{n+1} \in T x_{n}$ such that $\rho\left(x_{n}, x_{n+1}\right)<\eta^{n} \sigma$. Note that $\rho\left(x_{0}, x_{n+1}\right)$ $<\sigma\left(1+\eta+\cdots+\eta^{n}\right)<\sigma /(1-\eta)=d\left(x_{0}, T x_{0}\right) /(1-\eta)+\varepsilon$; in particular $x_{n+1} \in B$. In this way, we have a Cauchy sequence, which converges, say to $v$. Then $d\left(x_{0}, v\right) \leq d\left(x_{0}, T x_{0}\right) /(1-\eta)+\varepsilon$ so $v \in B$. The proof that $v \in \bar{T} v$ is similar to [10]: take $\gamma>0$ and a positive integer $n$. Then there is $y \in T v$ such that $\rho\left(x_{n}, y\right)<d\left(x_{n}, T v\right)+\gamma$ so

$$
\rho\left(x_{n}, y\right)<d\left(T x_{n-1}, T v\right)+\gamma \leq \eta \rho\left(x_{n-1}, v\right)+\gamma
$$


and

$$
d(v, T v) \leq \rho(v, y) \leq \rho\left(v, x_{n}\right)+\rho\left(x_{n}, y\right) \leq \rho\left(v, x_{n}\right)+\eta \rho\left(x_{n-1}, v\right)+\gamma .
$$

Letting $n \rightarrow \infty$ and $\gamma \rightarrow 0$, we see that $v \in \overline{T v}$.

We now turn to the proof of Theorem 1. We shall apply Lemma 1 to $\Omega=X$ with $\rho$ the usual metric induced by the norm. The inverse $U^{-1}$ of the multivalued function $U$ is defined by

$$
U^{-1} y=\{x \in X: y \in U x\} \quad(y \in Y) .
$$

By assumption each $U^{-1} y$ is non-empty. We will show that

$$
d\left(U^{-1} y_{1}, U^{-1} y_{2}\right) \leq k\left\|y_{1}-y_{2}\right\| \quad\left(y_{1}, y_{2} \in Y\right) .
$$

In fact, let $x_{1} \in U^{-1} y_{1}$. Since $U$ is $k$-open, there is $x \in X$ with $\|x\| \leq k\left\|y_{2}-y_{1}\right\|$ such that $y_{2}-y_{1} \in U x$. Then

$$
y_{2}=\left(y_{2}-y_{1}\right)+y_{1} \in u(x)+K+u\left(x_{1}\right)+K=u\left(x+x_{1}\right)+K=U\left(x+x_{1}\right)
$$

because $K$ is a convex cone. Therefore $x+x_{1} \in U^{-1} y_{2}$, and

$$
d\left(x_{1}, U^{-1} y_{2}\right) \leq \rho\left(x_{1}, x+x_{1}\right)=\|x\| \leq k\left\|y_{2}-y_{1}\right\| .
$$

Since $x_{1}$ is arbitrary in $U^{-1} y_{1},(3)$ is proved.

Now define $T$ on $D$ by $T w=U^{-1}(g(w))$ where $g(w):=u(z)-f(w)+u(w)$. By (1), we have, for all $w_{1}, w_{2} \in D$, that

$$
\left\|g\left(w_{1}\right)-g\left(w_{2}\right)\right\|=\left\|\left\{f\left(w_{2}\right)-u\left(w_{2}\right)\right\}-\left\{f\left(w_{1}\right)-u\left(w_{1}\right)\right\}\right\| \leq \eta / k\left\|w_{1}-w_{2}\right\| ;
$$

it follows from (3) that $d\left(T w_{1}, T w_{2}\right) \leq \eta\left\|w_{1}-w_{2}\right\|$. Moreover, since $g(0)=u(z)$, $z \in U^{-1}(u(z))=T 0$, we have

$$
d(z, T z) \leq d(T 0, T z) \leq \eta\|z-0\|=\eta\|z\| .
$$

By the Contraction Lemma, there exists $x \in B$ such that $x \in \overline{T x}$. Take a sequence $\left\{x_{n}\right\}$ in $T x$ convergent to $x$. Then $g(x) \in U\left(x_{n}\right)=u\left(x_{n}\right)+K$, that is,

$$
u(z)-f(x)+u(x) \in u\left(x_{n}\right)+K .
$$

Since $K$ is closed it follows that $u(z) \in f(x)+K$.

THEOREM 2. Let $C$ be a closed convex cone in $Y$, and $Q$ a subset of $Y$ such that $Q+C \subseteq Q$ and $\lambda Q \subseteq Q$ for all $\lambda \in[0,1]$. Let $f$ be a $C^{1}$-function at 0 from an open set in $X$ containing 0 into $Y$, with $f(0)=0$ and $f^{\prime}(0)=u$. Define $U$ by $U(x)=u(x)-C$ for all $x \in X$. If $U$ is onto $Y$, then $U^{-1}(Q)$ is contained in the tangent cone of $f^{-1}(Q)$ at 0 .

Proof. It is known that $U$ is $k$-open for some $k>0$ as noted before. Let $h \in$ $U^{-1}(q)$ with $\|h\|=1$ and $q \in Q$. Then $q \in U(h)=u(h)-C$ so $u(h) \in C+Q \subseteq Q$ 
and consequently $u(\lambda h) \in Q$ for all $\lambda \in[0,1]$. Take $\eta \in(0,1)$; then there exists $\xi>0$ such that $\left\|f^{\prime}(x)-u\right\| \leq \eta / k$ for all $x$ in $\xi B_{X}$ the $\xi$-ball with centre 0 in $X$. By the Mean Value Theorem, (1) of Theorem 1 holds with $D:=\xi B_{X}$. Take $\lambda>0$, small enough that $D$ contains the open ball with centre $\lambda h$ and radius $2 \eta \lambda /(1-\eta)$. Applying Theorem 1 there is $x \in X$ with $\|x-\lambda h\| \leq 2 \eta\|\lambda h\| /(1-\eta)$ such that $u(\lambda h) \in f(x)-C$, that is, $f(x) \in u(\lambda h)+C \subseteq Q+C \subseteq Q$.

Do the above for all $\eta=1 / n$ with integers $n>3$ and choose $\lambda=\lambda_{n}>0$ such that $\lambda_{n} \rightarrow 0$ as $n \rightarrow \infty$; we write $x_{n}$ for $x$ accordingly constructed above. Note that $x_{n} \neq 0, x_{n} \in f^{-1}(Q), x_{n} \rightarrow 0$ and

$$
\begin{aligned}
\left\|x_{n}\right\| x_{n}\left\|^{-1}-\lambda_{n} h\right\| \lambda_{n} h\left\|^{-1}\right\| & \leq 2\left\|x_{n}-\lambda_{n} h\right\|\left\|\lambda_{n} h\right\|^{-1} \\
& \leq 4 \eta /(1-\eta) \rightarrow 0 \text { as } n \rightarrow \infty,
\end{aligned}
$$

where we have used the elementary inequality $\|a\| a\left\|^{-1}-b \mid\right\| b\left\|^{-1}\right\| \leq$ $2\|a-b\|\|b\|^{-1}$ for non-zero elements in a normed space, which is true because

$$
\left\|(a\|b\|-a\|a\|-b|| a\|+a\| a \|)(\|a\|\|b\|)^{-1}|| \leq 2|| a\right\|\|b-a\|(\|a\| \| b||)^{-1} .
$$

Therefore $h$ is in the tangent cone of $f^{-1}(Q)$ at 0 .

REMARK. A related result has been given by Robinson [12, Corollary 2] where he considered the case $Q=C$. Applications of results of this type to Optimization Theory, have been given in [1], [2], [3], [4], [6], [9], [12] and [14].

\section{References}

[1] P. J. Bender, 'Nonlinear programming in normed linear spaces', J. Optimization Theory Appl. 24 (1978), 263-285.

[2] B. D. Craven, 'A generalization of Lagrange multipliers', Bull. Austral. Math. Soc. 3 (1970), 353-362.

[3] B. D. Craven, Mathematical programming and control theory, (Chapman and Hall, 1978).

[4] T. M. Flett, 'On differentiation in normed vector spaces', J. London Math. Soc. 42 (1967), 523-533.

[5] G. Jameson, Ordered linear spaces, (Springer-Verlag, 1970).

[6] D. Luenberger, Optimization by vector space method, (John Wiley, 1968).

[7] S. B. Nadler, Jr., 'Multi-valued contraction mappings', Pacific J. Math. 30 (1969), 475488.

[8] K. F. Ng, 'An open mapping theorem', Math. Proc. Cambridge Philos. Soc. 74 (1973), 61-66.

[9] K. F. Ng and D. Yost, 'Quasi-regularity in optimization', J. Austral. Math. Soc. Ser. A 41 (1986), 188-192.

[10] S. M. Robinson, 'Normed convex processes', Trans. Amer. Math. Soc. 174 (1972), 127-140.

[11] S. M. Robinson, 'An inverse-function theorem for a class of multivalued functions', Proc. Amer. Math. Soc. 41 (1973), 211-218.

[12] S. M. Robinson, 'Stability theory for systems of inequalities, Part II: differentiable nonlinear systems', SIAM J. Numer. Anal. 13 (1976), 497-513. 
[13] R. T. Rockafellar, 'Monotone processes of convex and concave type', Mem. Amer. Math. Soc. 77 (1967).

[14] J. Zowe and S. Kurcyusz, 'Regularity and stability for the mathematical programming problem in Banach spaces', Appl. Math. Optim. 5 (1979), $49-62$.

Department of Mathematics

The Chinese University of Hong Kong

Hong Kong 